\title{
Elliptic Curve Over SPIR Of Characteristic Two
}

\author{
Abdelhamid TADMORI \\ Departement of Mathematics \\ Faculty of science Mohamed first \\ University Oujda MOROCCO \\ atadmori@yahoo.fr
}

\author{
Abdelhakim CHILLALI \\ FST \\ USMBA, FES \\ MORROCO \\ chil2007@voila.fr
}

\author{
M'hamed ZIANE \\ Departement of Mathematics \\ Faculty of science Mohamed first \\ University Oujda MOROCCO \\ Ziane20011@yahoo.fr
}

\begin{abstract}
In [1] and [4] we defined the elliptic curve over the ring $\mathbb{F}_{3^{d}}[\varepsilon], \varepsilon^{2}=0$. In this work, we will study the elliptic curve over the ring $\mathbf{A}=\mathbb{F}_{2^{d}}[\varepsilon]$, where $d$ is a positive integer and $\varepsilon^{2}=0$. More precisely we will establish a group homomorphism between the abulia group $\left(\mathbf{E}_{a, b, c}\left(\mathbb{F}_{2^{d}}\right),+\right)$ and $\left(\mathbb{F}_{2^{d}},+\right)$.
\end{abstract}

\section{INTRODUCTION}

Let $\mathrm{d}$ be an integer, we consider the quotient ring

$$
\mathbf{A}=\frac{\mathbb{F}_{2^{d}}[X]}{\left(X^{2}\right)}
$$

where $\mathbb{F}_{2^{d}}$ is the finite field of order $2^{d}$.

Then the ring $\mathbf{A}$ is identified to the ring $\mathbb{F}_{2^{d}}[\varepsilon]$ with $\varepsilon^{2}=0$, ie:

$$
\mathbf{A}=\left\{a_{0}+a_{1} \cdot \varepsilon \mid a_{0}, a_{1} \in \mathbb{F}_{2^{d}}\right\} .
$$

We consider the elliptic curve over the ring $\mathbf{A}$ which is given by equation $Y^{2} Z+c X Y Z=X^{3}+a X^{2} Z+b Z^{3}$, where a,b,c are in $\mathbf{A}$ and $c^{6} b$ is invertible in $\mathbf{A}$, see [1] and [2].

\section{NOTATIONS}

Let a,b,c $\in \mathbf{A}$, such that $c^{6} b$ is invertible in $\mathbf{A}$. We denote the elliptic curve over $\mathbf{A}$ by $\mathbf{E}_{a, b, c}(\mathbf{A})$ and we write:

$$
\mathbf{E}_{a, b, c}(\mathbf{A})=\left\{[X: Y: Z] \in \mathbb{P}_{2}(\mathbf{A}) \mid Y^{2} Z+c X Y Z=X^{3}+a X^{2} Z+b Z^{3}\right\} .
$$

If $b_{0}, c_{0} \in \mathbb{F}_{2^{d}} \backslash\{0\}$ and $a_{0} \in \mathbb{F}_{2^{d}}$, we also write:

$\mathbf{E}_{a_{0}, b_{0}, c_{0}}\left(\mathbb{F}_{2} d\right)=\left\{[X: Y: Z] \in \mathbb{P}_{2}\left(\mathbb{F}_{2}\right) \mid Y^{2} Z+c_{0} X Y Z=X^{3}+a_{0} X^{2} Z+b_{0} Z^{3}\right\}$

III. Classification of elements of $\mathbf{E}_{a, b, c}(\mathbf{A})$

Let $[X: Y: Z] \in \mathbf{E}_{a, b, c}(\mathbf{A})$, where $\mathrm{X}, \mathrm{Y}$ and $\mathrm{Z}$ are in $\mathbf{A}$. We have tow cases for $Z$ :

- $\mathrm{Z}$ invertible: then $[X: Y: Z]=\left[X Z^{-1}: Y Z^{-1}: 1\right]$ hence we take just [X:Y:1].

- Z non invertible: so $Z=z_{1} \varepsilon$, see [3] in this cases we have tow cases for $\mathrm{Y}$.

- If Y invertible: then $[X: Y: Z]=\left[X Y^{-1}: 1: Z Y^{-1}\right]$, so we just take $\left[X: 1: z_{1} \varepsilon\right] \in \mathbf{E}_{a, b, c}(\mathbf{A})$, then is verified the equation of

$$
\mathbf{E}_{a, b, c}(\mathbf{A}): Y^{2} Z+c X Y Z=X^{3}+a X^{2} Z+b Z^{3},
$$

so we can write:

$$
a=a_{0}+a_{1}
$$

$$
\begin{aligned}
b & =b_{0}+b_{1} \\
c & =c_{0}+c_{1} \\
X & =x_{0}+x_{1}
\end{aligned}
$$

- We have:

$z_{1} \varepsilon+\left(c_{0}+c_{1} \varepsilon\right) \cdot\left(x_{0}+x_{1} \varepsilon\right) \cdot z_{1} \varepsilon=\left(x_{0}+x_{1} \varepsilon\right)^{3}+\left(a_{0}+a_{1} \varepsilon\right) \cdot\left(x_{0}+x_{1} \varepsilon\right)^{2} \cdot z_{1} \varepsilon+\left(b_{0}+b_{1} \varepsilon\right) \cdot z_{1}^{3} \varepsilon^{3}$,

which implies that

$$
z_{1} \varepsilon+\left(c_{0}+c_{1} \varepsilon\right) \cdot\left(x_{0} z_{1} \varepsilon\right)=x_{0}^{3}+\left(x_{0}^{2} x_{1}+a_{0} x_{0}^{2} z_{1}\right) \varepsilon
$$

So

$$
z_{1} \varepsilon+c_{0} x_{0} z_{1} \varepsilon=x_{0}^{3}+\left(x_{0}^{2} x_{1}+a_{0} x_{0}^{2} z_{1}\right) \varepsilon
$$

then

$$
\left(z_{1}+c_{0} x_{0} z_{1}\right) \cdot \varepsilon=x_{0}^{3}+\left(x_{0}^{2} x_{1}+a_{0} x_{0}^{2} z_{1}\right) \varepsilon
$$

since $(1, \varepsilon)$ is a base of the vector space $\mathbf{A}$ over $\mathbb{F}_{2^{d}}$ then $x_{0}=0$, so $X=x_{1} \varepsilon$ and $z_{1} \varepsilon=0$ (ie $z_{1}=0$ ) hence $\left[X: 1: z_{1} \varepsilon\right]=\left[x_{1} \varepsilon: 1: 0\right]$.

- If Y non invertible: then we have $Y=y_{1} \varepsilon$, so $x=x_{0}+x_{1} \varepsilon$ is invertible so we take $[X: Y: Z] \sim$ $\left[1: y_{1} \varepsilon: z_{1} \varepsilon\right]$, thus $1+a . z_{1} \varepsilon=0$, ie: $1+a_{0} \cdot z_{1} \varepsilon=0$, which is absurd

Proposition 1. Every element of $\mathbf{E}_{a, b, c}(\mathbf{A})$ is of the form $[X: Y: 1]$ or $[x \varepsilon: 1: 0]$, where $x \in \mathbb{F}_{2^{d}}$ and we write

$\mathbf{E}_{a, b, c}(\mathbf{A})=\left\{[X: Y: 1] \in \mathbb{P}_{2}(\mathbf{A}) \mid Y^{2}+c X Y=X^{3}+a X^{2}+b\right\} \bigcup\left\{[x \varepsilon: 1: 0] \mid x \in \mathbb{F}_{2^{d}}\right\}$.

\section{THE $\pi_{2}$ HOMOMORPHISM}

We consider the canonical projection $\pi$ defined by:

$$
\begin{aligned}
\pi: \mathbb{F}_{2^{d}}[\varepsilon] \longrightarrow \mathbb{F}_{2^{d}} \\
x_{0}+x_{1} \varepsilon \longmapsto x_{0}
\end{aligned}
$$

Lemma 2. $\pi$ is a morphism of rings.

Proof.. let $X=x_{0}+x_{1} \varepsilon$ and $Y=y_{0}+y_{1} \varepsilon$ then:

$$
\begin{gathered}
X+Y=x_{0}+y_{0}+\left(x_{1}+y_{1}\right) \varepsilon \\
X \cdot Y=\left(x_{0}+x_{1} \varepsilon\right) \cdot\left(y_{0}+y_{1} \varepsilon\right) \\
\quad=x_{0} \cdot y_{0}+x_{0} y_{1} \varepsilon+y_{0} x_{1} \varepsilon \\
=x_{0} \cdot y_{0}+\left(x_{0} y_{1}+y_{0} x_{1}\right) \varepsilon,
\end{gathered}
$$


so :

$$
\begin{aligned}
& \pi(X+Y)=\pi(X)+\pi(y) \\
& \pi(X \cdot Y)=\pi(X) \times \pi(Y),
\end{aligned}
$$

therefore $\pi$ is a morphism of rings.

Lemma 3. Let $[X: Y: Z] \in \mathbb{P}_{2}(\mathbf{A})$, where

$$
\begin{aligned}
& X=x_{0}+x_{1} \varepsilon \\
& Y=y_{0}+y_{1} \varepsilon \\
& Z=z_{0}+z_{1} \varepsilon \\
& a=a_{0}+a_{1} \varepsilon \\
& b=b_{0}+b_{1} \varepsilon \\
& c=c_{0}+c_{1} \varepsilon
\end{aligned}
$$

$$
\begin{aligned}
& \text { then }[X: Y: Z] \in \mathbf{E}_{a, b, c}(\mathbf{A}) \text { if and only if } \\
& y_{0}^{2} z_{0}+c_{0} x_{0} y_{0} z_{0}=x_{0}^{3}+a_{0} x_{0}^{2} z_{0}+b_{0} z_{0}^{3}
\end{aligned}
$$

$y_{0}^{2} z_{1}+c_{0} x_{0}\left(y_{0} z_{1}+y_{1} z_{0}\right)+y_{0} z_{0}\left(c_{0} x_{1}+c_{1} x_{0}\right)=a_{0} x_{0}^{2} z_{1}+b_{1} z_{0}^{3}+a_{1} x_{0}^{2} z_{0}+x_{0}^{2} x_{1}+b_{0} z_{0}^{2} z_{1}$

Proof.. Since $(1, \varepsilon)$ is a base of the vector space $\mathbf{A}$ over $\mathbb{F}_{2^{d}}$, and $[X: Y: Z] \in \mathbf{E}_{a, b, c}(\mathbf{A})$, then

$$
Y^{2} Z+c X Y Z=X^{3}+a X^{2} Z+b Z^{3},
$$

so after the compute, we find the result.

$\star$ Let $\pi_{2}$ the mapping defined by:

$$
\begin{gathered}
\mathbf{E}_{a, b, c}(\mathbf{A}) \stackrel{\pi_{2}}{\longrightarrow} \mathbf{E}_{a_{0}, b_{0}, c_{0}}\left(\mathbb{F}_{2^{d}}\right) \\
{[X: Y: Z] \longmapsto[\pi(X): \pi(Y): \pi(Z)]}
\end{gathered}
$$

We proof that the mapping $\pi_{2}$ is a surjective homomorphism of groups.

Theorem 4. Let $\mathbf{P}=\left[X_{1}: Y_{1}: Z_{1}\right]$ and $\mathbf{Q}=\left[X_{2}: Y_{2}: Z_{2}\right]$ tow points in $\mathbf{E}_{a, b, c}(\mathbf{A})$, and $\mathbf{P}+\mathbf{Q}=\left[X_{3}: Y_{3}: Z_{3}\right]$.

- If $\pi_{2}(\mathbf{P})=\pi_{2}(\mathbf{Q})$, then:

$X_{3}=X_{1} Y_{1} Y_{2}^{2}+X_{2} Y_{1}^{2} Y_{2}+c X_{2}^{2} Y_{1}^{2}+c^{2} X_{1} X_{2}^{2} Y_{1}+a X_{1}^{2} X_{2} Y_{2}+a X_{1} X_{2}^{2} Y_{1}+$ $a c X_{1}^{2} X_{2}^{2}+b X_{1} Y_{1} Z_{2}^{2}+b X_{2} Y_{2} Z_{1}^{2}+b c X_{1}^{2} Z_{2}^{2}+c^{2} b Y_{1} Z_{2}^{2} Z_{1}+c^{2} b Y_{2} Z_{1}^{2} Z_{2}+$ $c^{3} b X_{1} z_{2}^{2} z_{1}$

$Y_{3}=Y_{1}^{2} Y_{2}^{2}+c X_{2} Y_{1}^{2} Y_{2}+a c X_{1} X_{2}^{2} Y_{1}+a^{2} X_{1}^{2} X_{2}^{2}+b X_{1}^{2} X_{2} Z_{2}+$ $b X_{1} X_{2}^{2} Z_{1}+b c X_{1} Y_{1} Z_{2}^{2}+b c^{2} X_{1}^{2} Z_{2}^{2}+a b X_{2}^{2} Z_{1}^{2}+b c^{3} Y_{1} Z_{1} Z_{2}^{2}+b c^{4} X_{1} Z_{1} Z_{2}^{2}+$ $a b c^{2} X_{1} Z_{1} Z_{2}^{2}+a b c^{2} X_{2} Z_{1}^{2} Z_{2}+b^{2} Z_{1}^{2} Z_{2}^{2}$

$Z_{3}=X_{1}^{2} X_{2} Y_{2}+X_{1} X_{2}^{2} Y_{1}+Y_{1}^{2} Y_{2} Z_{2}+Y_{1} Y_{2}^{2} Z_{1}+c X_{1}^{2} X_{2}^{2}+c Y_{1}^{2} X_{2} Z_{2}+$ $c^{2} X_{1}^{2} Y_{2} Z_{2}+a X_{1}^{2} Y_{2} Z_{2}+a X_{2}^{2} Y_{1} Z_{1}+c^{3} X_{1}^{2} X_{2} Z_{2}+a c X_{1} X_{2}^{2} Z_{1}+b Y_{1} Z_{1} Z_{2}^{2}+$ $b Y_{2} Z_{1}^{2} Z_{2}+b c X_{1} Z_{1} Z_{2}^{2}$

- If $\pi_{2}(\mathbf{P}) \neq \pi_{2}(\mathbf{Q})$, then:

$x_{3}=X_{1} Y_{2}^{2} Z_{1}+X_{2} Y_{1}^{2} Z_{2}+c X_{1}^{2} Y_{2} Z_{2}+c X_{2}^{2} Y_{1} Z_{1}+a X_{1}^{2} X_{2} Z_{2}+a X_{1} X_{2}^{2} Z_{1}+$ $b X_{1} z_{1} z_{2}^{2}+b X_{2} Z_{1}^{2} z_{2}$

$Y_{3}=X_{1}^{2} X_{2} Y_{2}+X_{1} X_{2}^{2} Y_{1}+Y_{1}^{2} Y_{2} Z_{2}+Y_{1} Y_{2}^{2} Z_{1}+c^{2} X_{1}^{2} Y_{2} Z_{2}+c^{2} X_{2}^{2} Y_{1} Z_{1}+$ $a X_{1}^{2} Y_{2} Z_{2}+a X_{2}^{2} Y_{1} Z_{1}+a c X_{1}^{2} X_{2} Z_{2}+a c X_{1} X_{2}^{2} Z_{1}+b Y_{1} Z_{1} Z_{2}^{2}+b Y_{2} Z_{1}^{2} Z_{2}+$ $b c X_{1} z_{1} z_{2}^{2}+b c X_{2} z_{1}^{2} z_{2}$

$z_{3}=X_{1}^{2} X_{2} Z_{2}+X_{1} X_{2}^{2} Z_{1}+Y_{1}^{2} Z_{2}^{2}+Y_{2}^{2} Z_{1}^{2}+c X_{1} Y_{1} Z_{2}^{2}+c X_{2} Y_{2} Z_{1}^{2}+a X_{1}^{2} Z_{2}^{2}+$ $a X_{2}^{2} Z_{1}^{2}$

Proof.. Using the explicit formulas in W.Bosma and H.Lenstra article , see [5], we prove the theorem.
Lemma 5. The mapping $\pi_{2}$ is a surjective homomorphism of groups

Proof.. The formula of lemma(3) means that $\pi_{2}([X: Y$ : $Z])=\left[x_{0}: y_{0}: z_{0}\right]$, and $\left[x_{0}: y_{0}: z_{0}\right] \in \mathbf{E}_{a_{0}, b_{0}, c_{0}}\left(\mathbb{F}_{2^{d}}\right)$, so $\pi_{2}$ is well defined.

$\pi_{2}$ is surjective: let $\left[x_{0}: y_{0}: z_{0}\right] \in \mathbf{E}_{a_{0}, b_{0}, c_{0}}\left(\mathbb{F}_{2^{d}}\right)$ we will show that $\left[x_{0}: y_{0}: z_{0}\right]$ have an antecedent

$$
[x: y: z] \in \mathbf{E}_{a, b, c}(\mathbf{A})
$$

- Case 1: $z_{0}=0$, then $\left[x_{0}: y_{0}: z_{0}\right]=[0: 1: 0]$ and we just take $[X: Y: Z]=[0: 1: 0]$.

- Case 2: $z_{0} \neq 0$, then $\left[x_{0}: y_{0}: z_{0}\right]=\left[z_{0}^{-1} x_{0}: z_{0}^{-1} y_{0}: 1\right]$ so we just take $\left[x_{0}: y_{0}: 1\right]$

so we will find an antecedent $[X: Y: Z]$ of $\left[x_{0}: y_{0}: 1\right]$ of the form

$$
\left[x_{0}+x_{1} \varepsilon: y_{0}+y_{1} \varepsilon: 1\right] \text {, }
$$

from the formulas of the lemma (3) we have:

$$
y_{0}^{2}+c_{0} x_{0} y_{0}=x_{0}^{3}+a_{0} x_{0}^{2}+b_{0},
$$

and

$$
c_{0}\left(x_{0} y_{1}+y_{0} x_{1}\right)+c_{1} x_{0} y_{0}=a_{1} x_{0}^{2}+x_{0}^{2} x_{1}+b_{1},
$$

there is three sub-cases:

- Case 2,1: $x_{0} \neq 0$, then we just take

$$
[X: Y: Z]=\left[x_{0}: y_{0}+\left(c_{0} x_{0}\right)^{-1} \cdot\left(a_{1} x_{0}^{2}+c_{1} x_{0} y_{0}+b_{1}\right) \varepsilon: 1\right],
$$

because $c^{6} b$ is invertible so $c_{0} \neq 0$

- Case 2,2: $y_{0} \neq 0$, then,we just take

$$
[X: Y: Z]=\left[\left(c_{0} y_{0}\right)^{-1} \cdot b_{1} \varepsilon: y_{0}: 1\right]
$$

- Case 2,3: $y_{0}=0$ and $x_{0}=0$ then we have $b_{0}=0$ absurd because $c^{6} b$ is invertible ie $b_{0} \neq 0$ and $c_{0} \neq 0$

$\pi_{2}$ is an homomorphism: we just use the theorem(4) and lemma(2)

\section{Lemma 6.}

$$
[x \varepsilon: 1: 0]+[y \varepsilon: 1: 0]=[(x+y) \varepsilon: 1: 0]
$$

Proof.. We have $\pi_{2}([x \varepsilon: 1: 0])=\pi_{2}([y \varepsilon: 1: 0])$, so by applying the formula in theorem (4)we have:

$$
X_{3}=(x+y) \varepsilon, Y_{3}=1+c y \varepsilon \text { and } Z_{3}=0,
$$

So

$[x \varepsilon: 1: 0]+[y \varepsilon: 1: 0]=[(x+y) \varepsilon: 1+c y \varepsilon: 0]=[(x+y) \varepsilon: 1: 0]$

Lemma 7. The mapping

$$
\begin{gathered}
\mathbb{F}_{2^{d}} \stackrel{\theta}{\longrightarrow} \mathbf{E}_{a, b, c}(\mathbf{A}) \\
x \longmapsto[x \varepsilon: 1: 0]
\end{gathered}
$$

is an injective morphism of groups. 
Proof.. $\theta$ is well defined because

$$
[x \varepsilon: 1: 0] \in \mathbf{E}_{a, b, c}(\mathbf{A}),
$$

see proposition (1) and from the lemma (6)we have:

$\theta(x+y)=[(x+y) \varepsilon: 1: 0]=[x \varepsilon: 1: 0]+[y \varepsilon: 1: 0]=\theta(x)+\theta(y)$,

then $\theta$ is a morphism.

$\bullet \theta$ is injective (evidently)

\section{Lemma 8.}

$$
\operatorname{Ker}\left(\pi_{2}\right)=\theta\left(\mathbb{F}_{2^{d}}\right)
$$

Proof.. Evidently we have: $\theta\left(\mathbb{F}_{2^{d}}\right) \subseteq \operatorname{Ker}\left(\pi_{2}\right)$, now let,

$\mathbf{P}=[X: Y: Z]=\left[x_{0}+x_{1} \varepsilon: y_{0}+y_{1} \varepsilon: z_{0}+z_{1} \varepsilon\right] \in \operatorname{Ker}\left(\pi_{2}\right)$, implies that $\pi_{2}(\mathbf{P})=\left[x_{0}: y_{0}: z_{0}\right]=[0: 1: 0]$, implies that $\mathbf{P}=\left[x_{1} \varepsilon: 1: z_{1} \varepsilon\right] \in \mathbf{E}_{a, b, c}(\mathbf{A})$ and from the proposition (1) we have:

$\mathbf{P}=[x \varepsilon: 1: 0] \in \theta\left(\mathbb{F}_{2^{d}}\right)$, ie: $\operatorname{Ker}\left(\pi_{2}\right) \subseteq \theta\left(\mathbb{F}_{2^{d}}\right)$, hence

$\operatorname{Ker}\left(\pi_{2}\right)=\theta\left(\mathbb{F}_{2^{d}}\right)$

From lemmas (5), (7) and (8) we deduce the following corollary:

Corollary 9. The sequence

$$
0 \longrightarrow \operatorname{Ker}\left(\pi_{2}\right) \stackrel{i}{\longrightarrow} \mathbf{E}_{a, b, c}(\mathbf{A}) \stackrel{\pi_{2}}{\longrightarrow} \mathbf{E}_{a_{0}, b_{0}, c_{0}}\left(\mathbb{F}_{2^{d}}\right) \longrightarrow 0
$$

is a short exact sequence which defines the group extension $\mathbf{E}_{a, b, c}(\mathbf{A})$ of $\mathbf{E}_{a_{0}, b_{0}, c_{0}}\left(\mathbb{F}_{2^{d}}\right)$ by $\operatorname{Ker}\left(\pi_{2}\right)$, where $i$ is the canonical injection.

\section{CRYPTOGRAPHIC APPLICATION}

Let $\mathbf{E}_{a, b, c}(\mathbf{A})$ an elliptic curve over $A$ and $P \in \mathbf{E}_{a, b, c}(\mathbf{A})$ of order $l$. We will use the subgroup $\langle P\rangle$ of $\mathbf{E}_{a, b, c}(\mathbf{A})$ to encrypt messages, and we denote $G=\langle P\rangle$.

1) Coding of elements of $G$ We will give a code to each element $Q=m P$ where $m \in\{1,2, . ., l\}$ defined as it follows:

if $Q=\left[x_{0}+x_{1} \varepsilon: y_{0}+y_{1} \varepsilon: Z\right]$ where $x_{i}, y_{i} \in A$ for $i=0$ or 1 and $Z=0$ or 1 . We set:

$$
\begin{aligned}
& x_{i}=c_{0 i}+c_{1 i} \alpha \\
& y_{i}=d_{0 i}+d_{1 i} \alpha
\end{aligned}
$$

where $\alpha$ is primitive root of an irreducible polynomial of degree 2 over $\mathbb{F}_{2}$ and $c_{i j}, d_{i j} \in \mathbb{F}_{2}$.

Then we code $Q$ as it follows:

If $Z=1$ then: $Q=c_{00} c_{10} c_{01} c_{11} d_{00} d_{10} d_{01} d_{11} 1$

If $Z=0$ then: $Q=00 c_{01} c_{11} 10000$

2) Example Let $a=0, b=1+\varepsilon$ and $c=1$.

So the elliptic curve $\mathbf{E}_{a, b, c}(\mathbf{A})$ has 32 elements: $\{[0$ : $1: 0],[1: \varepsilon: 1],[1: 1+\varepsilon: 1],[\alpha:(\alpha+1) \epsilon: 1],[\alpha:$ $\alpha+(\alpha+1) \varepsilon: 1],[\epsilon: 1: 0],[\varepsilon: 1: 1],[\varepsilon: 1+\varepsilon: 1],[\varepsilon:$ $1+\alpha \varepsilon: 1],[\varepsilon: 1+(\alpha+1) \varepsilon: 1],[\alpha \varepsilon: 1: 0],[(\alpha+1) \varepsilon:$ $1: 0],[1+\varepsilon: 0: 1],[1+\varepsilon: 1+\varepsilon: 1],[1+\alpha \varepsilon:(\alpha+1) \varepsilon:$ $1],[1+\alpha \varepsilon: 1+\varepsilon: 1],[1+(\alpha+1) \varepsilon: \alpha \epsilon: 1],[1+(\alpha+1) \varepsilon:$ $1+\varepsilon: 1],[\alpha+1: \alpha \varepsilon: 1],[\alpha+1: \alpha+1+\alpha \varepsilon: 1],[\alpha+\varepsilon:$ $\alpha: 1],[\alpha+\varepsilon: \varepsilon: 1],[\alpha+\alpha \varepsilon: 0: 1],[\alpha+\alpha \varepsilon: \alpha+\alpha \varepsilon:$
1], $[\alpha+(\alpha+1) \varepsilon: \alpha \varepsilon: 1],[\alpha+(\alpha+1) \varepsilon: \alpha+\varepsilon:$ 1], $[\alpha+1+\varepsilon: \epsilon: 1],[\alpha+1+\varepsilon: \alpha+1: 1],[\alpha+1+\alpha \varepsilon:$ $(\alpha+1) \varepsilon: 1],[\alpha+1+\alpha \varepsilon: \alpha+1+\varepsilon: 1],[\alpha+1+(\alpha+1) \varepsilon:$ $0: 1],[\alpha+1+(\alpha+1) \varepsilon: \alpha+1+(\alpha+1) \varepsilon: 1]\}$ Let

$P=[\alpha+1+(\alpha+1) \varepsilon: \alpha+1+(\alpha+1) \varepsilon: 1]=111111111$, we have:

$$
2 P=[1+\alpha \varepsilon+\varepsilon: 1+\varepsilon: 1]=101110101
$$

$$
3 P=[\alpha+\varepsilon: \varepsilon: 1]=011000101
$$$$
4 P=[\varepsilon: 1+\varepsilon: 1]=001010101
$$$$
5 P=[\alpha+(\alpha+1) \varepsilon: \alpha+\varepsilon: 1]=011101101
$$

$$
6 P=[1+\alpha \varepsilon: \alpha \varepsilon+\varepsilon: 1]=1001001111
$$

$$
7 P=[\alpha+1: \alpha \varepsilon: 1]=110000011
$$

$$
8 P=[\varepsilon: 1+\alpha \varepsilon: 0]=010010011
$$

$$
\begin{gathered}
9 P=[\alpha+1: \alpha+1+\alpha \varepsilon: 1]=1100110111 \\
10 P=[1+\alpha \varepsilon: 1+\varepsilon: 1]=100111001 \\
11 P=[\alpha+(\alpha+1) \varepsilon: \alpha \varepsilon: 1]=011100011 \\
12 P=[\varepsilon: 1: 1]=010010001 \\
13 P=[\alpha+\varepsilon: \alpha: 1]=011001001 \\
14 P=[1+\alpha \varepsilon+\varepsilon: \alpha \varepsilon: 1]=110100011 \\
15 P=[\alpha+1+\alpha \varepsilon+\varepsilon: 0: 1]=111100001
\end{gathered}
$$

and $16 P=[0: 1: 0]=000010000$

so, $G=\{111111111,101110101,011000101,001010101$, 011101101, 1001001111, 110000011, 010010011, 1100110111, 100111001, 011100011, 010010001, 011001001, 110100011, $111100001,000010000\}$.

\section{CONCLUSION}

In this work we have studed the elliptic curve over the ring $\mathbf{A}=\frac{\mathbb{F}_{2 d}[X]}{\left(X^{2}\right)}$, precisely we have established the short exact sequence that defines the group extension $\mathbf{E}_{a, b, c}(\mathbf{A})$ of $\mathbf{E}_{\pi_{2}(a), \pi_{2}(b), \pi_{2}(c)}\left(\mathbb{F}_{2^{d}}\right)$ by $\operatorname{Ker}\left(\pi_{2}\right)$, and we have gived an example of cryptography over this ring. 


\section{REFERENCES}

[1] Abdelhakim chillali, the j-invariant over $\mathbf{E}^{n}$. Int.j.Open problems Compt. Math.,Vol.5,No.4,December 2012,ISSN 1998-6262; Copyright ICSRS Publication,WWW.i-csrs.org,pp.106-111 (2012).

[2] Abdelhakim chillali, Elliptic curve over ring, International Mathematical Forum,Vol.6,no.31,2011 pp.1501-1505

[3] Abdelhakim chillali, Cryptography over elliptic curve of the ring $\mathbb{F}_{q}[\varepsilon], \varepsilon^{4}=0$ World Academy of science Engineering and Technology,78 (2011),pp.848-850

[4] M.H. Hassib and A. Chillali, Example of cryptography over the ring $\mathbb{F}_{3 d}[\varepsilon], \varepsilon^{2}=0$, Latest trends in Applied Informatics and Computing, p.71-73, ISBN 978-1-61804-130-2, (2012).

[5] W.Bosma and H.Lenstra, Complete system of two addition laws for eliptic curved, Journal of Number theory (1995).

[6] J. Lenstra, H.W, Elliptic curves and number-theoretic algorithms, Processing of the International Congress of Mathematicians, Berkely, California, USA,(1986).

[7] M.VIRAT. courbe elliptique sur un anneau et applications cryptographiques, These Docteur en Sciences, Nice-Sophia Antipolis. (2009)

[8] N.KOBLITZ. Elliptic Curve Cryptosystems, Mathematics of Computation.48,203,209,(1987).2,6,21,37

[9] R.LERCIER. Algorithmique de courbes elliptiques dans les corps finis, $\mathrm{PhD}$ thesis, Ecole polytechnique. juin (1997).

[10] J.H.SILVERMAN. The Arithmetic of Elliptic curves, Graduate Texts in Mathematcs. Springer.Volume 106(1985).2,19,20,21

[11] J.H.SILVERMAN. Advanced Topics in the Arithmetic of Elliptic curves, Graduate Texts in Mathematcs. Volume 151, Springer,(1994).

[12] V.CHANDRASEKARAN, N.NAGARAJAN. Novel Approach Design of Elliptic curve Cryptography Implementation in VLSI,RECENT ADVANCES in NETWORKING, VLSI and SIGNAL PROCESSING. www.wseas.us/e-library/conferences/2010/Cambridge/.../ICNVS-17.pdf

[13] Kapil A. Gwalani, Omar Elkeelany. Design and Evaluation of Hardware Accelerator for Elliptic Curve Cryptography Point Multiplication, RECENT ADVANCES IN APPLIED MATHEMATICS AND COMPUTATIONAL AND INFORMATION SCIENCES - Volume II.www.wseas.us/e-library/conferences/2009/houston/.../AAMCIS229.pdf

\section{Creative Commons Attribution License 4.0 (Attribution 4.0 International, CC BY 4.0)}

This article is published under the terms of the Creative Commons Attribution License 4.0

https://creativecommons.org/licenses/by/4.0/deed.en US 